\title{
CARACTERIZAÇÃO DA MIRMECOFAUNA EM ESTABELECIMENTOS LIGADOS À ÁREA DA SAÚDE NO MUNICÍPIO DE BANDEIRANTES, PR
}

\author{
J.S.S. Rando ${ }^{1}$, L.S. Matsumoto ${ }^{2}$, G.V.Silva ${ }^{3}$, A.F.A. Quirino ${ }^{4}$, R.E. Haddad ${ }^{4}$
}

${ }^{1}$ Universidade Estadual do Norte do Paraná, Departamento de Produção Vegetal, CP 261, CEP 86360-000, Bandeirantes, PR, Brasil. E mail: jael@ffalm.br

\section{RESUMO}

\begin{abstract}
As formigas urbanas são consideradas um problema grave na saúde pública pela capacidade de ocupar todos os ambientes e por serem vetores mecânicos de micro-organismos. O presente trabalho teve por objetivo verificar as espécies que ocorrem em estabelecimentos ligados a área da saúde, bem como os microrganismos veiculados por essas espécies. Em um total de 1.629 coletas realizadas de outubro a dezembro de 2007em um hospital, na Secretaria de Saúde e em um laboratório de análises clinicas, utilizando-se iscas atrativas não tóxicas, as seguintes espécies foram registradas foram registradas: Tapinoma melanocephalum (Fabricius, 1793); Monomorium pharaonis (Linnaeus, 1758); Paratrechina fulva (Mayr, 1862); Pheidole triconstricta Forel, 1886; Pheidole sp.1; Pheidole sp.2; Pheidole sp.3; Pheidole sp.4; Camponotus atriceps (Fr.Smith, 1858); Brachymyrmex sp.; Dorymyrmex sp. Como micro-organismos constatou-se a presença de bactérias: Staphylococcus sp.; Serratia sp.; Klebsiella sp.; Escherichia coli; Salmonella sp., Pseudomonas sp. e de fungos Cladosporium sp.; Fusarium sp.; Trichophyton sp.; Aspergillus sp. e Penicillium sp. além de leveduras.
\end{abstract}

PALAVRAS-CHAVE: Insecta, formigas urbanas, micro-organismo, vetor mecânico.

\section{ABSTRACT}

CHARACTERIZATION OF MYRMECOFAUNA IN ESTABLISHMENTS LINKED TO THE HEALTHSECTORINTHECOUNTY OFBANDEIRANTES, PR, BRAZIL. Urban ants are considered a serious problem for public health due to their capacity to occupy all environments and for their being mechanical vectors of microorganisms. The present work was aimed to verify the occurrence of ant species at a hospital, at the General Office of Health, and at a laboratory of clinical analysis, in the county of Bandeirantes, PR, Brazil. A total of 1,629 collections were made in the period from October to December 2007, using nonpoisonous baits. The species recorded were: Tapinoma melanocephalum (Fabricius, 1793); Monomorium pharaonis (Linnaeus, 1758), Paratrechina fulva (Mayr, 1862), Pheidole triconstricta Forel, 1886, Pheidole sp. 1, Pheidole sp. 2, Pheidole sp. 3, Pheidole sp. 4, Camponotus atriceps (Fr. Smith, 1858), Brachymyrmex sp., and Dorymyrmex sp. The microorganisms included the bacteria Staphylococcus sp., Serratia sp., Klebsiella sp., Escherichia coli, Salmonella sp., and Pseudomonas sp.; the fungi Cladosporium sp., Fusarium sp., Trichophyton sp., Aspergillus sp. and Penicillium sp.; as well as yeasts.

KEY WORDS: Insecta, tramp ant species, microorganism, mechanical vector.

\section{INTRODUÇÃO}

As formigas são os animais dominantes na maioria dos ecossistemas terrestres (WILSON, 1987).

As formigas são denominadas de benéficas quando associadas à reciclagem de nutrientes, polinização e dispersão de sementes de plantas e aeração do solo, entre outras atividades (LuNA et al.,2004). Também são citadas comoagentes de controlebiológico, reduzindo no agroecossistema a população de pragas (QuEIROz et al., 2006), sendo particularmente efetivas no controle de larvas que vivem no solo, pupas e adultos de diversas espécies de insetos (DE BACH, 1977).

No Brasil, das cerca de 2.000 espécies de formigas, 20 a 30 são consideradas pragas urbanas (BuENO; CAMPOS-FARINHA, 1999).

O estabelecimento de formigas invasoras é quase sempre associado a ambientes instáveis, incluindo

\footnotetext{
${ }^{2}$ Universidade Estadual do Norte do Paraná, Departamento de Patologia, Bandeirantes, PR, Brasil.

${ }^{3}$ Acadêmica do Curso de Ciências Biológicas -UENP- Campus Luiz Meneghel, Bandeirantes, PR, Brasil.

${ }^{4}$ Acadêmicas do Curso de Enfermagem- UENP- Campus Luiz Meneghel, Bandeirantes, PR, Brasil.
} 
aqueles criados pela atividade humana (GuLLAN; CRANSTON, 2007). As formigas urbanas, de modogeral, são onívoras percorrendo grandes distâncias em busca de alimentos, visitando latas de lixo, caixas de gordura, dejetos, saída de esgotos eo ambiente domiciliar (THYSSEN et al., 2004).

As formigas são bem sucedidas em diversos ambientes urbanos, principalmente porque podem utilizar uma grande variedade de alimentos que são avaliados por elas de acordo com sua riqueza nutricional. Elas dispõem de plantas e material animal, mas muitas espécies preferem alimentos ricos em açúcar, carboidratos, óleos e proteínas (ROBINSON, 1996).

Muitas espécies se tornam indesejadas quando ocupam museus, fábricas de alimentos, padarias, restaurantes, equipamentos, estabelecimentos comerciais, instituições de pesquisa, residências, centrais telefônicas, equipamentos eletro-eletrônicos, entre outros (Bueno; CAMpos-Farinha 1999; Oliveira; CAMPOS-FARINHA, 2005). GAULD; Bolton (1988) relatam que, com o aumento na utilização de aquecedores centrais em apartamentosna Inglaterra, houve um incremento no estabelecimento e frequência de infestações de formigas "andarilhas", destacando Monomorium pharaonis; M. floricola e Tapinoma melanocephalum, que são atraídas pela temperatura alta e uniforme gerada por esses aparelhos que favorecem a sobrevivência das colônias no inverno.

Consideradas um problema na saúde pública, as formigas se dispersam nos hospitais e centros de saúde, alimentando-se de material descartado, fragmentos de sangue em ataduras, material em decomposição, restos de medicamentos, percorrendo frestas, ralos, frequentando ambientes limpos e contaminados. Nos hospitais, uma grande variedade de alimentos está disponível por toda parte nas cozinhas, nos cafés, em carrinhos que transportam refeições e máquinas delanches ou refeições leves (BURRUS, 2004).

Vários autores são unânimes no reconhecimento da capacidade de vecção de micro-organismos patogênicos pelas formigas em instituições ligadas à área de saúde humana, relacionando as bactérias Pseudomonas sp., Enterobacter sp., Escherichia sp. e Serratia sp. e fungos filamentosos, entre outros, considerados nocivos ao homem. Alertam ainda pela necessidade premente de estudos sobre a diversidade de formigas urbanas e sua associação com microorganismos (ROBINSON, 1996; WetLer et al., 2004; RODOvalHo et al., 2007; TANAKA et al., 2007; FALKKOPERECK et al., 2007). Assim, esta pesquisa teve como objetivo identificar as espécies de formigas e levantar os micro-organismos veiculados pelo conjunto delas, em três estabelecimentos da saúde em Bandeirantes, Paraná.

\section{MATERIALEMÉTODOS}

A pesquisa foi desenvolvida no Município de Bandeirantes, Paraná, localizado a $21^{\circ} 15^{\prime}$ de Latitude Sul e $50^{\circ} 23^{\prime}$ de Longitude Oeste e altitude média de $440 \mathrm{~m}$. Escolheu-se três estabelecimentos ligados à área da saúde, que apresentam grande fluxo de pessoas no períododiurno. Foram analisados um hospital privado com área de $2.600 \mathrm{~m}^{2}$, a Secretaria da Saúde com 1.550 $\mathrm{m}^{2}$ e um laboratório de análises clínicas com $188 \mathrm{~m}^{2}$.

Duranteoperíodo de desenvolvimento da pesquisa (outubro a dezembro de 2007) os estabelecimentos não sofreram dedetização tanto nas áreas internas quanto externas.

Nas visitas, registrava-seem todos os locais as temperaturas com um termômetro de máxima e mínima, além de observar qualquer ocorrência que pudesse interferir na atividade das formigas, tais como lavagem dopiso, aplicação de desinfetantes, atendimentos como vacinação, injeção de medicamentos e curativos, entre outros. Foram definidos dez locais de coleta no laboratório de análises clínicas (salas de coleta, esterilização e análises), vinte e três na Secretaria da Saúde (setores de atendimento médico especializado, pronto atendimento e atendimento materno infantil) e dezessetelocais no hospital (setores de atendimento ambulatorial, internamento, centro cirúrgicoe de serviços). Para a captura das formigas foram utilizadas iscas atrativas não tóxicas, constituídas de pedaços de $2 \mathrm{~cm}$ de canudinhos de refrigerante e recheadas na mesma proporção com uma mistura de fígado bovino desidratado, mel e bolo de abacaxi (ZARZuela et al., 2002). As iscas eram fixadas com fita adesiva e dispostas em três pontos por local de coleta. As coletas eram feitas três vezes por semana, colocando-se as iscas às 12 horas e retirando-as às 14 horas. Devidoà restrição do horário de funcionamento dos dois primeiros estabelecimentos, as avaliações noturnas ocorreram somente no hospital, em dezessete locais e no horário das 21 e 23 horas. Para a coleta, utilizou-se um pincel com cerdas finas embebidas em álcool para retirar as formigas que estavam em contato com as iscas e/ou nas trilhas, transferindo-as para frascos contendo álcool $70 \%$, identificados por códigos, nas etiquetas dos locais e dos três pontos (no chão atrás da porta, na parede cerca de $20 \mathrm{~cm}$ abaixo da janela e próximo a tomadas ou interruptores elétricos). Em uma das avaliações semanais, as formigas capturadas eram colocadas em frascos contendo o meio de cultura ágar nutriente e, posteriormente, repicadas em meio ágar triptona de soja (TSA) para identificação de bactérias e ágar batata dextrose (ABD) para identificação de fungos filamentosos e leveduras, após incubação a $28^{\circ} \mathrm{C}$ por 48 horas.

As formigas foram identificadas utilizando-se as chaves de Bolton (1994) e Bueno; CAmpos-Farinha 
(1999) e enviadas para confirmação no Instituto Biológico de São Paulo. Os espécimes voucher das formigas encontram-se depositados na coleção de insetos do Laboratório de Pragas e Inimigos Naturais do Campus Luiz Meneghel/UENP.

Com os dados coletados foram determinadas as porcentagens das avaliações que apresentaram a ocorrência de formigas. Em cada avaliação efetuada, foram determinadas as espécies de formigas por local de coleta, em cada um dos estabelecimentos.

\section{RESULTADOSEDISCUSSÂO}

Olevantamento das espécies de formigas resultou em 1.629 coletas. Durante as visitas anotava-se a temperatura com auxílio de um termômetro de máxima emínima em todos os locais. A temperatura média nos dias de condução desta pesquisa variou de $21,3^{\circ} \mathrm{C}$ a $33,4^{\circ} \mathrm{C}$ como se apresenta na Tabela1. Entretanto, esta variação não interferiu no número delocais onde ocorreram as formigas, nos três estabelecimentos.

No posto de enfermagem do hospital (Tabela 2), independente do período de coleta, predominou a espécie Tapinoma melanocephalum (Fabricius, 1793). Nesse local as formigas estavam sempre presentes na pia alimentando-se de resíduos ou respingos de medicamentos como xarope, soro etc., formando uma fileira em direção a um ponto de tomada de energia elétrica na parede, onde provavelmentenidificavam.

Na recepção, somente no período diurno coletaram-se formigas $T$. melanocephalum, o que pode ser devido ao grande fluxo de pessoas para atendimento nos consultórios, e também ao consumo deágua, café e outros alimentos na sala de espera. Situação semelhante foi verificada por Вiсно et al. (2007) quando atribuíram a esses fatores a maior abundância tanto de espécies como de espécimes em hospitais e postos de saúde em Bagé, RS.

As atividades frequentes na cozinha permitiram a coleta de T. melanocephalum Brachymyrmex sp.ePheidole sp.2, nas paredes e próximo às janelas. A presença de uma dispensa para alimentos favorecia as atividades dessas formigas e sua coleta durante o dia e à noite.

Paratrechina fulva (Mayr, 1862) apresentou maior ocorrência nas coletas noturnas, tendo sido registrada uma única vez durante o dia. Entretanto, Soliset al. (2007), buscando conhecer aspectos da biologia de outra espécie desse gênero, observaram que $P$. longicornis (Latreille, 1802) foi mais ativa forrageando no período diurno. WeTterer et al. (1999) atribuíram as altas densidades dessa espécie à associação com altas densidades de homópteros que liberam secreções açucaradas, importante fonte de carboidratos para as formigas.

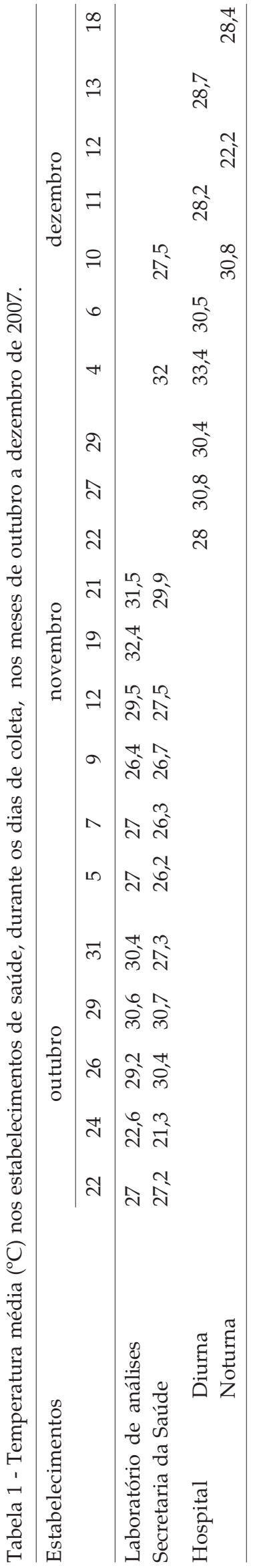


Tabela 2 - Porcentagem da ocorrência de formigas, em três iscas por local, nas visitas diurnas e noturnas ao hospital entre outubro e dezembro de 2007.

\begin{tabular}{|c|c|c|c|c|}
\hline Local & $\%$ diurna & espécie & $\%$ noturna & espécie \\
\hline Pronto socorro & 14 & $\mathrm{~b}$ & 33 & $\mathrm{a}, \mathrm{d}$ \\
\hline Farmácia & 0 & & 33 & $\mathrm{~d}$ \\
\hline Consultório & 0 & & 0 & \\
\hline Unidade de terapia intensiva & 0 & & 0 & \\
\hline Expurgo & 0 & & 0 & \\
\hline Posto de enfermagem & 100 & $\mathrm{a}, \mathrm{d}$ & 67 & a \\
\hline Apartamentos & 14 & $\mathrm{a}$ & 33 & $a, b, d$ \\
\hline Expurgo centro cirúrgico & 0 & & 33 & $\mathrm{a}$ \\
\hline Berçário 1 e 2 & 0 & & 0 & \\
\hline Cozinha & 71 & $\mathrm{a}, \mathrm{e}$ & 67 & $\mathrm{e}, \mathrm{h}, \mathrm{b}$ \\
\hline Lavanderia (área limpa) & 0 & & 33 & $\mathrm{a}, \mathrm{g}$ \\
\hline Lavanderia (área suja) & 43 & $\mathrm{a}, \mathrm{g}$ & 67 & $d, h, g, j$ \\
\hline Recepção & 100 & $\mathrm{a}$ & 0 & \\
\hline Necrotério & 14 & $\mathrm{j}$ & 67 & $c, f, h, i$ \\
\hline Centro cirúrgico & 0 & & 0 & \\
\hline Lixo contaminado & 0 & & 0 & \\
\hline
\end{tabular}

$\mathrm{a}=$ T. melanocephalum $; \mathrm{b}=P$. fulva $; \mathrm{c}=$ M. pharaonis $; \mathrm{d}=$ C. atriceps.; $\mathrm{e}=$ Brachymyrmex $\mathrm{sp}$;

$\mathrm{f}=$ Pheidole sp.4; $\mathrm{g}=$ Pheidole sp.3; $\mathrm{h}=$ Pheidole sp.2; $\mathrm{i}=$ Pheidole sp. $1 ; \mathrm{j}=$ Dorymyrmex $\mathrm{sp}$.

Pheidole sp.3 ocorreu somentena lavanderia, sendo três coletas diurnas (43\%) e em apenas uma noturna (33\%), dados estes semelhantes aos apresentados por ZARZUELA et al. (2002) que apontaram, em um hospital em Sorocaba, SP, a ocorrência de Pheidolesp. somente na lavanderia, nidificando próximo a uma fresta localizada no chão sob umajanela. Já as morfoespécies 1,2 e 4 de Pheidole foram coletadas no necrotério, no chão atrás da porta e abaixo da janela, no período noturno.

Monomorium pharaonis (Linnaeus,1758) éconsiderada uma espécie de interior de ambientes, mas saem para forragear externamente nas edificações a busca deinsetos mortos. Neste trabalho essa espécie ocorreu próximo à janela de um pátio externo. Oı et al. (1994) também relataram maior frequência dessas formigas em cartões adesivos colocados na janela. Esses dados reforçam a preocupação de diversos autores quanto ao risco que essa espécie representa para a saúde pública por transportar micro-organismos patogênicos (TANAKA et al., 2007; NiCKERSONetal., 2007). BuRRus (2004) comparou a preferência alimentar de $M$. pharaonis em material de descarte biológico tais como sangue, plasma, dextrose, solução salina e outros líquidos usados no cuidado dos pacientes, e concluiu que essa espécie é capaz de consumir especialmente dextrose a $5 \%$ no estado líquido e uma dieta suplementar desidratada.

O gênero Camponotus é constituído por espécies arborícolas e terrícolas, muitas possuem alta capacidade de invasão e adaptação para interagir com outros organismos (RAmos et al., 2003). Camponotus atriceps (Fr. Smith, 1858) foi coletada uma única vez durante o dia no posto deenfermagem, porém, à noite, foi capturada nas iscas colocadas sob a janela na farmácia, lavanderia (área suja), pronto-socorro e apartamentos, ratificando OLIVEIRA; CAMPOS-FARINHA (2005), que citaram entre outras características o hábito noturno dessa espécie.

Durante o dia coletou-se, nonecrotério eà noitena lavanderia (área suja), espécies de Dorymyrmex. A área externa a esse local é utilizada como depósito de material de construção usado nas reformas de ampliação do hospital, tais como areia, tijolos, entre outros, que favoreciam, provavelmente, a nidificação de algumas espécies. Embora não tenha sido escolhido como local de avaliação, observou-se, em uma sala que provisoriamente servia como depósito de papéis inutilizados, várias espécies do gênero Camponotus nas gavetas, arquivos, entre as folhas e nas pilhas de documentos antigos e material descartado.

Os dados das coletas no laboratório de análises clínicas encontram-se na Tabela 3. Eles registram que não houve diferença entre os locais de deposição das iscas.M. pharaonis esteve presenteem $100 \%$ das coletas, T. melanocephalum em $91 \%$ e Brachymyrmex sp. (9\%)em uma única vez atrás da porta no corredor de saída. SILVA; LOECK (1999) relataram que essa última espécie está entre as oito espécies de formigas mais frequentes nointerior de residências em Pelotas, RS. Já os habitantes de Maringá, PR, não consideraram essa espécie incô-moda, talvez por elas se associarem a plantas e seus insetos sugadores, que as mantém na parte externa dos domicílios (OliveIra; CAMPOS-FARINHA, 2005). 
Tabela 3 - Porcentagem da ocorrência de formigas, em três iscas por local, no laboratório de análises clínicas entre outubro e dezembro de 2007.

\begin{tabular}{lcc}
\hline Local & \% de ocorrência & Espécie \\
\hline Sala de espera & 91 & $\mathrm{a}, \mathrm{b}$ \\
Sala de coleta 1 & 91 & $\mathrm{a}, \mathrm{b}$ \\
Corredor de saída & 100 & $\mathrm{a}, \mathrm{b}, \mathrm{c}$ \\
Sala ginecológica & 45 & $\mathrm{a}, \mathrm{b}$ \\
Sala de lavagem e esterilização & 91 & $\mathrm{~b}$ \\
Depósito & 91 & $\mathrm{~b}$ \\
Cozinha & 91 & $\mathrm{~b}$ \\
Laboratório de análise & 91 & $\mathrm{~b}$ \\
Sala de preparo de reagentes & 82 & $\mathrm{~b}$ \\
Sala de microbiologia & 55 & $\mathrm{~b}$
\end{tabular}

$\mathrm{a}=$ T. melanocephalum; $\mathrm{b}=$ M. pharaonis; $\mathrm{c}=$ Brachymyrmex $\mathrm{sp}$.

Tabela 4 - Porcentagem da ocorrência de formigas, em três iscas por local, na Secretaria de Saúde entre outubro e dezembro de 2007.

\begin{tabular}{lll}
\hline Local & $\%$ de ocorrência & Espécie
\end{tabular}

Atendimento médico especializado

Sala de dispensação 33

Vigilância em saúde $\quad 75$

$\begin{array}{ll}\text { Refeitório } & 100\end{array}$

Expurgo

Sala de esterilização $\quad 0$

Sala de inalação $\quad 83$

$\begin{array}{ll}\text { Sala de injeção } & 75\end{array}$

Pronto atendimento

Cantina externa $\quad 92$

Sala de triagem $\quad 83$

$\begin{array}{ll}\text { Agendamento } & 75\end{array}$

Sala de odontologia $\quad 33$

$\begin{array}{ll}\text { Laboratório de análises } & 25\end{array}$

$a, b$
$a$
$a$
$a, c, d$

a,c,

(n)

Atendimento materno infantil

Sala de espera

Administração

$58 \quad a, b$

Dispensa $\quad 25$

$\begin{array}{ll}\text { Sala de triagem } & 67\end{array}$

Consultório ginecológico $\quad 33$

Sala de odontologia bebê clínica $\quad 42$

Consultório pediátrico 0

$\begin{array}{ll}\text { Copa } & 83\end{array}$

Sala de vacinas $\quad 83$

$\begin{array}{ll}\text { Sala de puericultura } & 17\end{array}$ 
nesse levantamento (Tabela 4). O gênero Pheidole é o mais abundante de todos os gêneros de formigas, em númerodecolôniaseindivíduos (WILSON,1986). Nesta pesquisa Pheidole sp.2 foi capturada no consultório ginecológico, na dispensa e no refeitório, sempre no ponto abaixo da janela. Na parede do refeitório e na sala de expurgo, coletou-se também Pheidole triconstricta Forel, 1886.

Segundo CAMPOS-FARINHA et al. (2002), apesar de $P$. fulva ser considerada uma espécie nativa, são poucas as informações sobre sua biologia no Brasil. Apresentaram ninhos pouco estruturados propensos a migração ao menor sinal de perturbação, com populações unicoloniais e alta agressividade, características que podem ter contribuído para a coleta apenas uma única vez, na sala de odontologia, em trilha se dirigindo a um ponto de tomada de energia elétrica de uso geral. SiLVA; LOECK (1999) e WETLER et al. (2004) registraram essa espécie também em residências.

Segundo Costa et al. (2006), as formigas são vetores mecânicos de varias espécies de micro-organismos, sendo considerados um dos fatores importantes na infecção hospitalar.

Neste trabalho encontraram-se as espécies de bactériascomoStaphylococcussp.,Pseudomonas sp., Klebsiella sp., Escherichiacoli, Salmonella sp.e Serratia sp. MENEZES etal.(2007) relataram queosúltimos quatrogêneros de enterobactérias estão associados às infecções hospitalares como patógeno oportunista, podendo causar distúrbiosintestinais, infecções dotrato urinário, pneumonias e septicemias. Micro-organismos como os Staphylococcus sp. são bactérias comumente encontradas na flora normal de indivíduos saudáveis. Porém, em condições de imunodepressão, podem provocar doenças que vão de uma simples infecção cutânea até infecções sistêmicas graves como pneumonia, endocarditeeatésepticemia (SANTOSetal.,2007). Foram encontrados, entre os fungos, as espécies de Cladosporium sp., Fusarium sp., Trichophyton sp.; Aspergillus sp. e leveduras.

\section{AGRADECIMENTOS}

Os autores agradecem à Dra. Ana Eugênia de Carvalho Campos, do Instituto Biológico deSão Paulo, pela contribuição na identificação das espécies.

\section{REFERÊNCIAS}

BICHO, C.L.; BRANCÃO, M.L.C.; PIRES, S. M. Mirmecofauna (Hymenoptera, Formicidae) em hospitais e postos de saúde no município de Bagé, RS. Arquivos do Instituto Biológico, São Paulo, v.74, n.4, p.373377, 2007.
BOLTON, B. Identification guide to the ant genera of the world.Cambridge: Harvard University Press, 1994. 222p.

BUENO, O.; CAMPOS-FARINHA, A.E.C. As formigas domesticas. In: MARICONI, F.A.M. (Coord.). Insetos: e outros invasores de residências. Piracicaba: FEALQ, 1999. cap.6, p.135-180.

BURRUS, R.G. Pharaoh ant consumption of fluids used in hospital environments. 2004.122p. Thesis (Master of Science) - University of Florida, Florida, 2004.

CAMPOS-FARINHA, A.E.C.; BUENO, O.C.; CAMPOS, M.C.B.; KATO, L.M. As formigas urbanas no Brasil: retrospecto. O Biológico, São Paulo, v.64, n.2, p.129-133, 2002.

COSTA, S.B. da; PELLI, A.; CARVALHO, G.P. de; OLIVEIRA, A.G.; SILVA, R. da; TEIXEIRA, M.M.; MARTINS, E.; TERRA, A.P.S.; R.E.M.; OLIVIRA, C.C.B. de; MORAIS, C.A. de Formigas como vetores mecânicos de microrganismos no Hospital Escola da Universidade Federal do Triângulo Mineiro. Revista da Sociedade Brasileira de Medicina Tropical, v.39, n.6, p.527-529, 2006.

DE BACH, P. Lucha biológica contra los enemigos de las plantas. Versión española de: Manuel Arroyo Varela y Candido Santiago Alvarez. Castilló: Mundi-Prensa, 1977. 386p. Título original: Biological control by natural enemies.

FALCK-KOPERECK, V.; DECKER, E.B.; DILLI, M.; VIANNA, A.D.; VIANNA, E.E.S. Levantamento parcial de espécies de formigas (Insecta: Formicidae) em Unidades Básicas de Saúde de Pelotas, RS. In: CONGRESSO DE INICIAÇÃO CIENTÍFICA DA UNIVERSIDADE FEDERAL DE PELOTAS, 16., 2007, Pelotas. Anais. Pelotas: UFPel, 2007. Disponível em: $<$ http:/ / www.ufpel.edu.br/cic/2007/cd/pdf/CB/ CB_00609.pdf $>$. Acesso em: 17 jun. 2008.

FOWLER, H. G.; FORTI, L. C.; BRANDÃO, C. R. F.; DELABIE, J.H.C.; VASCONCELOS, H.L. Ecologia nutricional de formigas, In: PANIZZI, A. R.; PARRA, J.R.P. (Ed.). Ecologia nutricional de insetos e suas implicações no manejo de pragas. São Paulo: Manole, 1991. cap.5, p.131-223.

GAULD, I.; BOLTON, B. (Ed.). The hymenoptera. New York: Oxford University Press. 1988. 332p.

GULLAN, P.J.; CRANSTON, P.S. Os insetos: um resumo de entomologia. 3.ed. Tradução de Sonia Maria Marques Hoenen. São Paulo: Roca, 2007.440p.

LUNA, E.; SOLIS, D. R.; BUENO, O. Monitoramento e identificação de formigas no refeitório e cantina da Escola Preparatória de Cadetes do Exército (ESPCEX), Campinas, SP. Arquivos do Instituto Biológico, São Paulo, v.71, p.222-224, 2004. Suplemento. Trabalho apresentado na REUNIÃO ANUAL DO INSTITUTO BIOLÓGICO, 17., 2004, São Paulo, SP. Resumo 148. 
MENEZES, E.A.; SÁ, K.M.; CUNHA, F.A.; ANGELO, M.R.F.; OLIVEIRA, I.R.N.; SALVIANO, M.N.C. Freqüência e percentual de suscetibilidade de bactérias isoladas em pacientes atendidos na Unidade de Terapia Intensiva do Hospital Geral de Fortaleza. Jornal Brasileiro de Patologia e Medicina Laboratorial, v.43, n.3, p.149-155, 2007.

NICKERSON, J.C.; HARRIS, D.L.; FASULO, T.R. Pharaoh ant, Monomorium pharaonis (LINNAEUS) (Insecta: Hymenoptera; Formicidae). EENY-290. Disponível em: <http:/ / creatures.ifas.ufl.edu.>. Acesso em: 25 jun. 2008.

OI, D.H.; VAIL, K.M.; WILLIAMS, D.F.; BIEMAN, D.N. Indoor and outdoor foraging locations of pharaoh ants (Hymenoptera: Formicidae) and control strategies using bait stations. Florida Entomologist, v.77, n.1, p.8592, 1994.

OLIVEIRA, M.F. de; CAMPOS-FARINHA, A.E.C. Formigas urbanas no município de Maringá, PR, e suas implicações. Arquivos do Instituto Biológico, São Paulo, v.72, n.1, p.33-39, 2005.

QUEIROZ, J.M. ALMEIDA, F.S. PEREIRA, M.P.S. Conservação da biodiversidade e o papel das formigas (Hymenoptera: Formicidae) em agroecossistemas. Floresta e Ambiente, v.13, n. 2, p.37-45, 2006.

RAMOS, L.S.; BONETTIFILHO, R.Z.; DELABIE, J.H.C.; LACAU, S.; SANTOS, M.F.S.dos; NASCIMENTO, I.C.; MARINHO, C.G.S. Comunidade de formigas (Hymenoptera: Formicidae) de serrapilheira em áreas de cerrado "strictu sensu" em Minas Gerais. Lundiana, v.4, n.2, p.95-102, 2003.

ROBINSON, W.H. Urban entomology: insect and mite pests in the human environment. 5.ed. London: Chapman \& Hall, 1996. 430p.

RODOVALHO, C.M.; SANTOS, A.L.; MARCOLINO, M.T.; BONETTI, A.M.; BRANDEBURGO, M.A.M. Urban ants and transportation of nosocomial bacteria. Neotropical Entomology, v.36, n.3, p.454-458, 2007.

SANTOS, A.L.; SANTOS, D.O.; FREITAS, C.C.; FERREIRA, B.L.A.; AFONSO, I.F.; RODRIGUES, C.R.; CASTRO, H.C.

Staphylus aureus: visitando uma cepa de importância hospitalar. Jornal Brasileiro de Patologia e Medicina Laboratorial, v.43, n.6, p.413-423, 2007.

SILVA, E.J.E.; LOECK, A.E. Ocorrência de formigas domiciliares (Hymenoptera: Formicidae) em Pelotas, RS. Revista Brasileira de Agrociência, v.5, n.3, p.220-224, 1999.
SOLIS, D.R.; BUENO, O.C.; MORETTI, T.C.; SILVS, T.F. da. Observações sobre a biologia da formiga invasora Paratrechina longicornis (Latreille, 1802) (Hymenoptera, Formicidae) em ambiente urbano brasileiro. Revista Brasileira de Zoociências, v.9, n.1, p.75-80, 2007.

TANAKA, I.I.; VIGGIANI, A. M. F. S.; PERSON, O.C. Bactérias veiculadas por formigas em ambiente hospitalar. Arquivos Médicos do ABC, v.32, n.2, p.60-63, 2007.

THYSSEN, P.J.; MORETTI, T.C.; UETA, M.T.; RIBEIRO, O.B. O papel de insetos (Blattodea, Diptera e Hymenoptera) como possíveis vetores mecânicos de helmintos em ambiente domiciliar e peridomiciliar. Cadernos de Saúde Pública, v.20, n.4, p.1096-1102, 2004.

WETLER, R.M.C.; CARVALHO, E.S.; CHAVES, N.A.; DELABIE, H.C. FONTANA, R. Formigas como vetor de propagação bacteriana: uma comparação entre formigas coletadas do campus da UESC e de um hospital público de Ilhéus. In: SEMINÁRIO DE INICIAÇÃO CIENTÍFICA DA UNIVERSIDADE ESTADUAL SANTA CRUZ, 10., 2004, Ilhéus, BA. Anais. Ilhéus: 2004. p.155-156.

WETTERER, J.K.; MILLER, S.E.; WHEELER, D.E.; OLSON, C.A.; POLHEMUS, D.A.; PITTS, M.; ASHTON, I.W.; HIMLER, A.G.; YOSPIN, M.M.; HELMS, K.R.; HARKEN, E.L.; GALLAHER, J.; DUNNING, C.E.; NELSON, M.; LITSINGER, J.; SOUTHERN,A.; BURGESS, T.L. Ecological dominance by Paratrechina longicornis (Hymenoptera: Formicidae), an invasive tramp ant, in biosphere 2. Florida Entomologist, v.83, n.3, p.381-388, 1999.

WILSON, E. O. The defining traits of fire ants and leaf cutting ants. In: LOEFGREEN, C.S.; VANDER MEER, R.K. (Ed.). Fire ants and leaf-cutting ants: biology and management. Boulder: Westview Press, 1986. chap.1. p.1-9.

WILSON, E.O. Causes of ecological success: the case of the ants. Journal of Animal Ecology, v.56, p.1-9, 1987.

ZARZUELA, M.F.M.; RIBEIRO, M.C.C.; CAMPOSFARINHA, A.E.C. Distribuição de formigas urbanas em um hospital da Região Sudeste do Brasil. Arquivos do Instituto Biológico, São Paulo, v.69, n.1, p.85-87, 2002.

Recebido em 9/7/08

Aceito em 20/5/09 